Article

\title{
Gas-Phase and Microsolvated Glycine Interacting with Boron Nitride Nanotubes. A B3LYP-D2* Periodic Study
}

\author{
Albert Rimola * and Mariona Sodupe \\ Departament de Química, Universitat Autònoma de Barcelona, 08193 Bellaterra, Catalonia, Spain; \\ E-Mail: mariona.sodupe@uab.cat \\ * Author to whom correspondence should be addressed; E-Mail: albert.rimola@uab.cat; \\ Tel.: +34-935-812-173; Fax: +34-935-812-477.
}

Received: 9 May 2014; in revised form: 29 May 2014 / Accepted: 30 May 2014 /

Published: 18 June 2014

\begin{abstract}
The adsorption of glycine (Gly) both in gas-phase conditions and in a microsolvated state on a series of zig-zag $(n, 0)$ single-walled boron nitride nanotubes (BNNTs, $n=4,6,9$ and 15 ) has been studied by means of B3LYP-D2* periodic calculations. Gas-phase Gly is found to be chemisorbed on the $(4,0),(6,0)$ and $(9,0)$ BNNTs by means of a dative interaction between the $\mathrm{NH}_{2}$ group of Gly and a $\mathrm{B}$ atom of the BNNTs, whose computed adsorption energies are gradually decreased by increasing the tube radius. On the $(15,0)$ BNNT, Gly is found to be physisorbed with an adsorption driving force mainly dictated by $\pi$-stacking dispersion interactions. Gly adsorption in a microsolvated environment has been studied in the presence of seven water molecules by progressively microsolvating the dry Gly/BNNT interface. The most stable structures on the $(6,0),(9,0)$ and $(15,0)$ BNNTs present the Gly/BNNT interface fully bridged by the water solvent molecules; i.e., no direct contact between Gly and the BNNTs takes place, whereas on the $(4,0)$ BNNT the most stable structure presents a unique direct interaction between the $\mathrm{COO}^{-}$Gly group and a $\mathrm{B}$ atom of the nanotube. Further energetic analyses indicate that the $(6,0),(9,0)$ and $(15,0)$ BNNTs exhibit a low water affinity, which favors the Gly/water interactions upon BNNT coadsorption. In contrast, the $(4,0)$ BNNT has been found to show a large water affinity, bringing the replacement of adsorbed water by a microsolvated glycine molecule as an unfavorable process.
\end{abstract}

Keywords: DFT; periodic simulations; biomolecules; boron nitrides; adsorption; microsolvation 


\section{Introduction}

Bioconjugated nanostructured materials resulting from the coupling of biomolecules with inorganic nanomaterials including nanotubes, nanowires, nanoparticles and nanosheets have attracted much attention during the last years as they exhibit unique features derived from combining synergistically the properties of the interacting components. These exclusive physico-chemical properties render these materials as suitable substrates with potential applications in diverse biological- [1,2] and material-related [3] areas such as biocatalysis,[4,5], drug delivery [6-8], biosensing [9-13] and medical diagnostics $[14,15]$. The functionalities resulting from these biohybrid materials are largely mediated by the biomolecule/inorganic surface interactions, which in turn are dictated by the structure-specific binding properties of the two partners. Accordingly, precise knowledge on the interactions between the biomolecule and the inorganic components is of fundamental relevance.

Among the different nanostructured materials, boron nitride nanotubes (BNNTs) have been proposed to be suitable candidates to be combined with biomolecules [16]. BNNTs are isosteres and structurally similar to carbon nanotubes (CNTs), in which alternating B and N atoms substitute for C atoms. However, these two type of nanotubes exhibit different physico-chemical properties. Whereas CNTs exhibit metallic or semiconducting behavior, which moreover is strongly dependent on the tube diameter, helicity and concentric layers, BNNTs are electrical insulators with a band gap of $c a .5 .5 \mathrm{eV}$ regardless of the tube geometry features [17]. Moreover, at variance with the non-polar $\mathrm{C}-\mathrm{C}$ bonds in CNTs, the B-N bonds of BNNTs exhibit a certain polar character, the degree of which depends on the curvature of the nanotube. That is, the increase of the tube curvature induces the transformation of the $\mathrm{sp}^{2}$ hybrid character of the $\mathrm{B}$ and $\mathrm{N}$ atoms in large diameter BNNTs into a $\mathrm{sp}^{3}$ one in small diameter BNNTs. As recently shown by us [18], this has important consequences for the nature of interaction between functional molecules and the BNNTs walls; i.e., polar molecules strongly chemisorb on small radius BNNTs, whereas interaction of non-polar molecules are energetically more favourable when physisorbed on large radius BNNTs. Furthermore, unlike CNTs, which present an inherent cytotoxicity [19], BNNTs have been found to be nontoxic [20] due to their high chemical and structural stability and high oxidation resistance, which alongside their uniformity and stability in dispersion in solution [21] make them suitable for biomedical applications.

Different experimental studies have focused on the interaction of peptides and proteins with BNNTs, showing a natural affinity between the two conjugates, which allows a direct immobilization of proteins on the BNNTs [22] as well as the isolation of individual BNNTs through a novel pathway based on peptide wrapping [23]. Moreover, biofunctionalized BNNTs via glycine interaction are good reactant substrates to obtain polysaccharide-coated BNNTs under mild conditions, in which the role of glycine is crucial during the interfacial process. The interactions of DNA and RNA with BNNTs have also been addressed and exploited to obtain nematic ordered ensembles of BNNT [24]. Other works have been devoted to assess the cytotoxicity of BNNTs when in contact with cells. Chen et al. [20] concluded that pristine BNNTs are inherently non-cytotoxic in view of the non-altered growth of human embryonic kidney (HEK) cells when cultured with BNNTs. Similar results were found by Ciofani and coworkers, in which coated-BNNTs presented a good cytocompatibility with human cells [25-28]. However, Goldberg and coworkers more recently found that BNNTs are actually cytotoxic for cells present in the lung alveoli and for HEK, in which the discrepancies with the other 
works were discussed and suggested to be due to the different morphology and size distribution of the BNNTs tested and the different assay techniques [29].

Theoretical works, mainly based on density functional theory (DFT) methods, have also studied the interaction of biomolecules with boron nitride nanostructures, most studies being limited to biomolecule building blocks, (amino acids and DNA and RNA nucleobases) due to the demanding computational cost of these calculations. Works on the gas-phase interaction of nucleobases using the local density approximation (LDA) and generalized gradient approximation (GGA) DFT levels of theory showed that this depends on the individual polarizations of the nucleobases [30-32]. The interaction of BNNTs with glycine (Gly) among other different amines has been studied in the gas-phase revealing an affinity of the $\mathrm{BNNT}$ with the $\mathrm{NH}_{2}$ Gly group [33]. Study on the gas-phase interaction of the arginine (Arg), aspartic acid (Asp) and tryptophane (Trp) amino acids, with basic, acidic and aromatic side chain functionalities, respectively, at the LDA DFT level revealed that the binding is accompanied by charge transfer following the trend of Arg > Asp > Trp [34]. The binding of different biomolecules inside the cavity of BNNTs has also been studied at the LDA level [35]. The calculated weak interactions led the authors to suggest BNNTs to be suitable biological carriers due to the limited delivery kinetic barrier.

All these works focus on the intrinsic adsorption properties; i.e., they are limited to the gas-phase and, accordingly, solvation effects were not accounted for. Moreover, each work addresses the interaction of biomolecules with a particular BNNT. Since it has been shown that the tubular radius can modulate the adsorption properties of BNNTs [18], which is also applicable for biomolecules, the nature of interaction can significantly be different depending on the radius of the BNNT. Moreover, for the particular works addressing the interaction of amino acids, no conformational exploration to find out the most stable amino acid/BNNT adduct was performed (i.e., the initial amino acid conformation guesses were the most stable gas-phase structure), which is an important drawback due to the large conformational mobility of these molecules. In order to provide a more complete atomic-scale description of the interaction of amino acids with BNNTs, the present work reports a systematic periodic B3LYP-D2* study, using a hybrid functional and including dispersion corrections, on the interaction of Gly with different zig-zag $(n, 0)$ single-walled BNNTs $(n=4,6,9$ and 15) rendering nanotubes of different radius. Note that dispersive effects, not included in previous works, are expected to play a role in these systems. Moreover, with the aim to study in a more realistic way the interactions between biological systems and BNNTs, the very same Gly/BNNTs interaction study has been addressed considering a microsolvated environment modeled by the presence of seven water molecules. The effect of water has been analyzed from a structural and energetic point of view, with particular attention paid to the Gly/BNNT interface to determine whether the interaction is direct or bridged by the water molecules.

\section{Results and Discussion}

\subsection{Gas-Phase Interaction}

An exhaustive description relative to the modeling of the different BNNTs used in this work in their pristine state is available in a very recent work by us [18]. The BNNTs are automatically generated by 
the CRYSTAL code through the wrapping of a periodic boron nitride monolayer (hexagonal P3m 1 layer symmetry group) into cylinders of different radius and fully exploiting the symmetry operators of the nanotubes [36-40]. In the interest of the present work, it is worth mentioning that the calculated electrostatic potential maps indicate a prominent positive/negative valued potential region for the $(4,0)$ BNNT, which become progressively less pronounced for BNNT with increasing radius, until obtaining a practically shallow electrostatic potential for the $(15,0)$ BNNT. For the interaction with Gly, the geometry optimizations were carried out as 1D polymers within the P1 space group, in which the unit cell parameters have been enlarged twice to avoid lateral interactions between molecules of adjacent unit cells.

Figure 1. (a) B3LYP-D2* optimized structures of the different calculated adducts for glycine interacting with the considered boron nitride nanotubes (BNNTs) in the gas-phase; (b) B3LYP-D2* optimized structures for those complexes in which a spontaneous proton transfer occurs during the geometry optimization (see text). Distances in $\AA$ : bare values for the $(4,0)$ BNNT; values in parenthesis for the $(6,0)$ one; values in brackets for the $(9,0)$ one; and italic underlined values for the $(15,0)$ one.

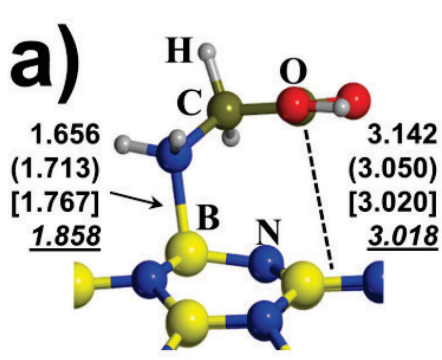

$\mathrm{BN} / \mathrm{NH}_{2}$

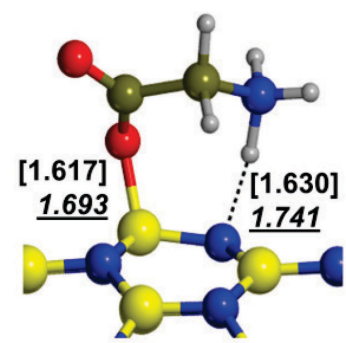

BN/zwitt

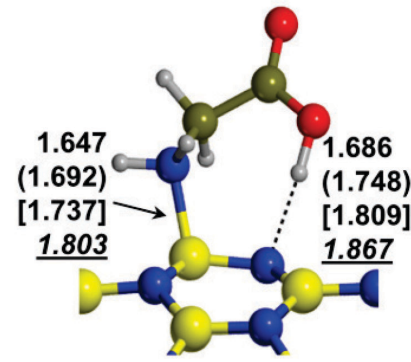

$\mathrm{BN} / \mathrm{NH}_{2}-\mathrm{OH}$

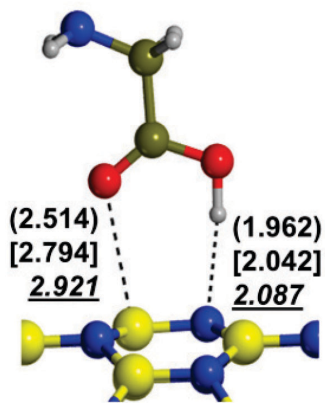

$\mathrm{BN} / \mathrm{COOH}-1$

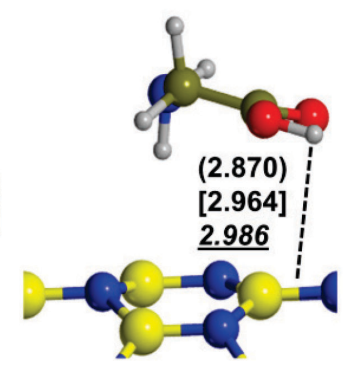

$\mathrm{BN} / \pi$

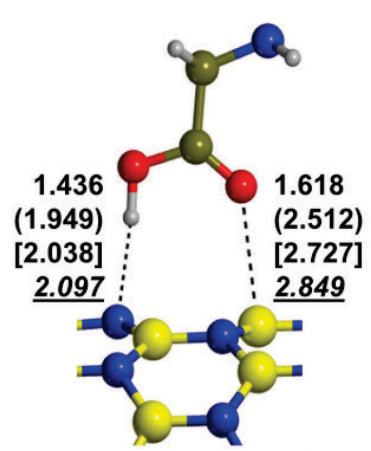

BN/COOH-2
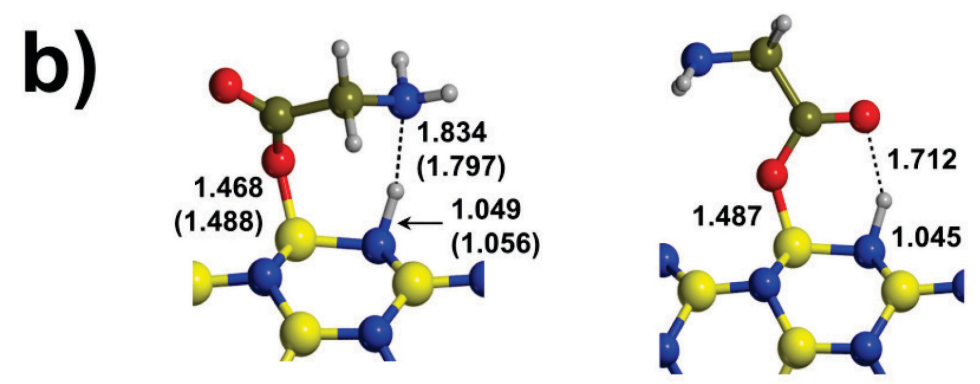
The different optimized adducts for the adsorption of Gly on the BNNTs are shown in Figure 1, whereas the calculated adsorption energies alongside the pure electronic and dispersion contributions are shown in Table 1 . The adsorption energies $\left(\Delta E_{\text {ads }}\right)$ per unit cell of the probe molecules with the BNNTs are computed as:

$$
\Delta E_{\mathrm{ads}}=E(\mathrm{Gly} / \mathrm{BNNT})-E(\mathrm{BNNT})-E_{\mathrm{m}}(\mathrm{Gly})
$$

where $E(\mathrm{Gly} / \mathrm{BNNT})$ is the energy of a fully relaxed unitary cell containing the BNNT in interaction with Gly, $E(\mathrm{BNNT})$ is the energy of a fully relaxed unitary cell of the BNNTs alone, and $E_{\mathrm{m}}(\mathrm{Gly})$ is the molecular energy of the free Gly.

For all the BNNT systems, six initial structural guesses were considered (see Figure 1a): (i) pure interaction between the $\mathrm{NH}_{2}$ group of Gly and one nanotube $\mathrm{B}$ atom (hereafter referred to as $\mathrm{BN} / \mathrm{NH}_{2}$ ); (ii) $\mathrm{BN} / \mathrm{NH}_{2}$ interaction plus $\mathrm{H}$-bonding between the Gly $\mathrm{OH}$ group and one nanotube $\mathrm{N}$ atoms (hereafter referred as $\mathrm{BN} / \mathrm{NH}_{2}-\mathrm{OH}$ ); (iii) and (iv) interaction between the Gly $\mathrm{CO}$ group and one nanotube $\mathrm{B}$ atom plus $\mathrm{H}$-bonding between the Gly $\mathrm{OH}$ group and one nanotube $\mathrm{N}$ atom, with the difference that the nanotube $\mathrm{B}$ and $\mathrm{N}$ atoms are chemically bonded or not (hereafter referred to as $\mathrm{BN} / \mathrm{COOH}-1$ and BN/COOH-2, respectively); (v) interaction of Gly in its zwitterionic form (hereafter referred to as $\mathrm{BN} / \mathrm{zwitt}$ ), in which the Gly $\mathrm{COO}^{-}$group interacts with one $\mathrm{B}$ atom and the $\mathrm{Gly} \mathrm{NH}_{3}{ }^{+}$is $\mathrm{H}$-bonded to one nanotube $\mathrm{N}$ atom; and (vi) interaction between Gly and the BNNTs purely through the $\pi$ system of the Gly $\mathrm{COOH}$ group (hereafter referred to as $\mathrm{BN} / \pi$ ).

Data reported in Table 1 clearly indicate that the most stable adduct for the $(4,0)$ BNNT is the $\mathrm{BN} / \mathrm{NH}_{2}-\mathrm{OH}$ whereas for the $(6,0)$ BNNTs both $\mathrm{BN} / \mathrm{NH}_{2}$ and $\mathrm{BN} / \mathrm{NH}_{2}-\mathrm{OH}$ are nearly degenerate. These complexes result from dative covalent interactions between the $\mathrm{Gly}_{\mathrm{NH}}$ group and the $\mathrm{B}$ atoms, which act as Lewis acid sites. These findings are consistent with the data reported for the interaction of these BNNTs with probe molecules, in which the interaction of $\mathrm{NH}_{3}$ with the $(4,0)$ and $(6,0)$ BNNTs was found to be the strongest one among all tested molecules [18]. The fact that the $\mathrm{BN} / \mathrm{NH}_{2}-\mathrm{OH}$ adduct becomes the most stable one for the Gly/BNNT(4,0) system is consistent with the large polar character of this nanotube, the $\mathrm{N}$ atoms acting as H-bonding acceptor groups. This is not in line with the most stable $\mathrm{BN} / \mathrm{NH}_{2}$ adduct found for the $(6,0)$ BNNT and is due to the weaker H-bonding acceptor character of the $\mathrm{N}$ nanotube atom when increasing the nanotube radius, which is reflected by an increase of the $\mathrm{H}$-bond distance in the $\mathrm{BN} / \mathrm{NH}_{2}-\mathrm{OH}$ adducts $(1.686$ and $1.748 \AA$ for the $(4,0)$ and $(6,0)$ BNNTs, see Figure 1a). In the same way, the B- $\mathrm{N}_{\mathrm{Gly}}$ bond lengths of the dative interactions in the $\mathrm{BN} / \mathrm{NH}_{2}$ and $\mathrm{BN} / \mathrm{NH}_{2}-\mathrm{OH}$ complexes also increase with increases in the nanotube radius due to the progressive decrease of the Lewis acceptor character of the $\mathrm{B}$ atom. Because of that, the calculated adsorption energy $\left(\Delta E_{\mathrm{ads}}\right)$ is more negative and larger for the most stable $\mathrm{BN} / \mathrm{NH}_{2}-\mathrm{OH}$ adduct of Gly/BNNT(4,0) than the most stable BN/NH $\mathrm{NH}_{2}$ adduct of Gly/BNNT(6,0) (i.e., -33.2 and $-18.9 \mathrm{kcal} \mathrm{mol}^{-1}$, respectively). The calculated energetic contributions; i.e., purely electronic and dispersion $\left(\Delta E_{\mathrm{el}}\right.$ and $\Delta E_{\mathrm{D}}$ values of Table 1 , respectively) indicate that Gly adsorption on the $(4,0)$ BNNT is largely dictated by the covalent dative interaction, whereas on the $(6,0)$ BNNT $\Delta E_{\text {el }}$ decreases in favor of dispersion. An analysis of the Mulliken charges ( $Q$ values of Table 2, only limited to the most stable Gly/BNNT complexes) confirms the formation of charge transfer complexes for both the Gly/BNNT(4,0) and Gly/BNNT(6,0) systems, the computed charge transfer values from Gly to the BNNTs being 0.30 e and 0.22 e, respectively. 
Table 1. Calculated adsorption energies $\left(\Delta E_{\text {ads }}\right)$, including the pure electronic energy contribution $\left(\Delta E_{\mathrm{el}}\right)$ and the contribution of dispersion $\left(\Delta E_{\mathrm{D}}\right)$. The relative electronic energies $\left(\Delta E_{\text {rel }}\right)$ for a given Gly/BNNT system are also included. Values in units of $\mathrm{kcal} \mathrm{mol}^{-1}$.

\begin{tabular}{llllll}
\hline System & Adduct & $\Delta \boldsymbol{E}_{\text {el }}$ & $\Delta \boldsymbol{E}_{\mathbf{D}}$ & $\Delta \boldsymbol{E}_{\text {ads }}$ & $\Delta \boldsymbol{E}_{\text {rel }}$ \\
\hline Gly/BNNT(4,0) & $\mathrm{BN} / \mathrm{NH}_{2}$ & -20.8 & -11.2 & -31.9 & 1.3 \\
& $\mathrm{BN} / \mathrm{NH}_{2}-\mathrm{OH}$ & -22.7 & -10.5 & -33.2 & 0.0 \\
& $\mathrm{BN} / \pi$ & - & - & - & - \\
& $\mathrm{BN} / \mathrm{zwitt}$ & -20.7 & -10.4 & -31.2 & 2.0 \\
& $\mathrm{BN} / \mathrm{COOH}-1$ & -24.6 & -7.1 & -31.7 & 2.2 \\
& $\mathrm{BN} / \mathrm{COOH}-2$ & -9.8 & -7.8 & -17.6 & 15.6 \\
\hline Gly/BNNT(6,0) & $\mathrm{BN} / \mathrm{NH}_{2}$ & -6.3 & -12.6 & -18.9 & 0.0 \\
& $\mathrm{BN} / \mathrm{NH}_{2}-\mathrm{OH}$ & -6.8 & -11.4 & -18.2 & 0.6 \\
& $\mathrm{BN} / \pi$ & +0.5 & -10.4 & -9.9 & 9.0 \\
& $\mathrm{BN} / \mathrm{zwitt}$ & -1.8 & -11.7 & -13.5 & 5.4 \\
& $\mathrm{BN} / \mathrm{COOH}-1$ & -3.3 & -6.3 & -9.6 & 8.0 \\
& $\mathrm{BN} / \mathrm{COOH}-2$ & -3.3 & -6.2 & -9.5 & 8.1 \\
\hline Gly/BNNT(9,0) & $\mathrm{BN} / \mathrm{NH}_{2}$ & +1.5 & -13.5 & -12.0 & 0.0 \\
& $\mathrm{BN} / \mathrm{NH}_{2}-\mathrm{OH}$ & +4.3 & -12.0 & -7.7 & 4.3 \\
& $\mathrm{BN} / \pi$ & +1.0 & -11.1 & -10.1 & 1.9 \\
& $\mathrm{BN} / \mathrm{zwitt}$ & +14.0 & -12.5 & +1.5 & 13.5 \\
& $\mathrm{BN} / \mathrm{COOH}-1$ & -2.6 & -6.0 & -8.6 & 3.4 \\
& $\mathrm{BN} / \mathrm{COOH}-2$ & -2.2 & -6.1 & -8.3 & 3.7 \\
\hline $\mathrm{BN} / \mathrm{NH}_{2}$ & +7.2 & -14.3 & -7.1 & 3.1 \\
& $\mathrm{BN} / \mathrm{NH}_{2}-\mathrm{OH}$ & +9.0 & -12.6 & -3.6 & 6.6 \\
$\mathrm{BN} / \pi$ & +1.5 & -11.7 & -10.2 & 0.0 \\
& $\mathrm{BN} / \mathrm{zwitt}$ & +20.7 & -13.1 & +7.6 & 17.8 \\
$\mathrm{BN} / \mathrm{COOH}-1$ & -1.4 & -6.1 & -7.5 & 2.7 \\
& $\mathrm{BN} / \mathrm{COOH}-2$ & -1.4 & -6.2 & -7.6 & 2.6 \\
\hline Gly/BNNT(15,0) & & & &
\end{tabular}

The most stable Gly/BNNT(15,0) adduct has been found to be the $\mathrm{BN} / \pi$ one. This is in perfect agreement with the EPM results, which point out the $(15,0)$ BNNT as a practically non-polar nanomaterial. Data reported in Table 1 clearly indicate that the binding mechanism involved in this adduct is mainly based on dispersive forces (calculated $\Delta E_{\text {ads }}$ is practically equal to $\Delta E_{\mathrm{D}} ;$ i.e., -10.2 and $-11.7 \mathrm{kcal} \mathrm{mol}^{-1}$, respectively) dictated by $\pi$-stacking interactions between the $\pi$ systems of the $\mathrm{COOH}$ group and the B-N hexagon rings of the $(15,0)$ BNNT. Because of the presence of only non-covalent interactions, $\Delta E_{\text {ads }}$ is less negative compared to Gly interaction on $(4,0)$ and $(6,0)$ BNNTs. It is worth mentioning that the very same $\mathrm{BN} / \pi$ complexes have also been calculated for the Gly/BNNT $(4,0)$ and $(6,0)$ systems, meaning that, in the former case, the structure collapses onto the $\mathrm{BN} / \mathrm{NH}_{2}$ complex, whereas for the latter case the calculated $\Delta E_{\text {ads }}$ is found to be $9.0 \mathrm{kcal} \mathrm{mol}^{-1}$ above the most stable one, due to the lower propensity of this BNNT to establish $\pi$-stacking interactions. 
Table 2. Muliken charge (Q) of Gly adsorbed on the BNNTs in the most stable adducts and respective calculated direct band gaps $\left(E_{\mathrm{g}}\right)$.

\begin{tabular}{llll}
\hline System & Adduct & $\mathbf{Q}(\mathbf{e})$ & $\boldsymbol{E}_{\boldsymbol{g}}(\mathbf{e V})^{\boldsymbol{a}}$ \\
\hline Gly/BNNT(4,0) & $\mathrm{BN} / \mathrm{NH}_{2}$ & 0.30 & 3.68 \\
& $\mathrm{BN} / \mathrm{NH}_{2}-\mathrm{OH}$ & 0.22 & 3.69 \\
\hline Gly/BNNT(6,0) & $\mathrm{BN} / \mathrm{NH}_{2}$ & 0.23 & 4.46 \\
& $\mathrm{BN} / \mathrm{NH}_{2}-\mathrm{OH}$ & 0.16 & 4.45 \\
\hline Gly/BNNT(9,0) & $\mathrm{BN} / \mathrm{NH}_{2}$ & 0.19 & 5.29 \\
& $\mathrm{BN} / \pi$ & -0.02 & 5.42 \\
\hline Gly/BNNT(15,0) & $\mathrm{BN} / \pi$ & -0.02 & 6.06 \\
\hline calculated direct band gaps for the pristine BNNTs: $3.67,4.42,5.42$ and $5.99 \mathrm{eV}$ for $(4,0),(6,0),(9,0)$ and \\
$(15,0)$, respectively.
\end{tabular}

The interaction of Gly with the $(9,0)$ BNNT is a frontier case between small radius (i.e., $(4,0)$ and $(6,0))$ and large radius (i.e., $(15,0))$ BNNTs. Although the $\mathrm{BN} / \mathrm{NH}_{2}$ adduct has been found to be the most stable one, the $\mathrm{BN} / \pi$ complex is the second most stable one lying $1.9 \mathrm{kcal} \mathrm{mol}^{-1}$ above. Calculated $\Delta E_{\text {ads }}$ values, however, can suffer from the basis set superposition error (BSSE). Indeed, upon correction, results indicate that these two complexes are nearly degenerate (BSSE-corrected $\Delta E_{\text {ads }}$ values being -9.4 and $-8.9 \mathrm{kcal} \mathrm{mol}^{-1}$ for the $\mathrm{BN} / \mathrm{NH}_{2}$ and $\mathrm{BN} / \pi$, respectively).

The interaction of Gly through the $\mathrm{COOH}$ group by means of a simultaneous $\mathrm{CO}-\mathrm{B}$ dative bond and a $\mathrm{OH} \cdots \mathrm{N}_{(\mathrm{BNNT})} \mathrm{H}$-bond has also been considered (see BN/COOH-1 and BN/COOH-2 adducts). Although none of the calculated complexes are the most stable ones, important structural and energetic features deserve to be mentioned. For the BN/COOH-1 adduct, Gly adsorption on the $(4,0)$ BNNT results in a spontaneous proton transfer from the Gly $\mathrm{COOH}$ group to the $\mathrm{N}$ nanotube atom, hence forming a COO-/BNNT- $\mathrm{H}^{+}$ion pair (see Figure $1 \mathrm{~b}$, structure of right). Such a proton transfer was already observed for the adsorption of $\mathrm{HCOOH}$ on the very same $(4,0) \mathrm{BNNT}$ and is attributed to the net charge transfer occurring from Gly to the BNNT, which induces an increase of the $\mathrm{COOH}$ acidity and the nanotube basicity up to the point of promoting the proton transfer to a nearby $\mathrm{N}$ atom of the nanotube. Moreover, for this adduct the $\mathrm{CO}-\mathrm{B}$ distance is significantly shorter than for the other BN/COOH-1 adducts (1.487 $\AA$ versus $2.514-2.921 \AA$, respectively), which results in a stronger interaction $\left(\Delta E_{\mathrm{ads}}=-31.7 \mathrm{kcal} \mathrm{mol}^{-1}\right.$ and $\approx-9.6--7.5 \mathrm{kcal} \mathrm{mol}^{-1}$, respectively). For the BN/COOH-2 complex on the $(4,0) \mathrm{BNNT}$, no proton transfer has been found; although the $\mathrm{OH}^{\cdots} \mathrm{N}_{(\mathrm{BNNT})} \mathrm{H}$-bond and the $\mathrm{CO}-\mathrm{B}$ dative bond are actually shorter than those present in the other BNNTs, in line with what has been described for the $\mathrm{BN} / \mathrm{COOH}-1$ cases. Interestingly, the difference between $\mathrm{BN} / \mathrm{COOH}-1$ and $\mathrm{BN} / \mathrm{COOH}-2$ is that in the former the $\mathrm{COOH}$ interaction occurs on $\mathrm{B}$ and $\mathrm{N}$ atoms that are chemically bonded to each other, whereas in the latter this is not the case. Accordingly, the fact that the spontaneous proton transfer only occurs in the former system seems to indicate that the charge transfer in enhanced by a cooperative effect between the $\mathrm{OH} \cdots \mathrm{N}_{(\mathrm{BNNT})}$ and the $\mathrm{CO}-\mathrm{B}$ interactions when the $\mathrm{B}$ and the $\mathrm{N}$ atoms are chemically bonded, which is in agreement with the larger and more negative calculated $\Delta E_{\text {ads }}$ values ( -31.7 and $-17.6 \mathrm{kcal} \mathrm{mol}^{-1}$ for BN/COOH-1 and BN/COOH-2, respectively).

Finally, it is worth mentioning that the interaction of Gly in its zwitterionic state has also been computed (BN/zwitt). On the $(4,0)$ and $(6,0)$ BNNTs, a spontaneous proton transfer from the $\mathrm{NH}_{3}{ }^{+}$ 
group to the $\mathrm{N}$ nanotube atom has been found, whereas for the $(9,0)$ and $(15,0)$ BNNTs the zwitterionic form is maintained. Consistently, calculated $\Delta E_{\text {ads }}$ values are negative for the two former adducts ( -31.2 and $-13.5 \mathrm{kcal} \mathrm{mol}^{-1}$, respectively), whereas for the two latter ones they have been found to be positive $\left(+1.5\right.$ and $+7.6 \mathrm{kcal} \mathrm{mol}^{-1}$, respectively) and, accordingly, are not stable complexes.

\subsection{Microsolvated Interaction}

Here, results on the interaction of Gly with the BNNTs in the presence of seven water molecules are reported. We have introduced seven water molecules since this is the minimum number of water molecules to have a relatively complete first-solvation shell of Gly upon adsorption; i.e., three water molecules interacting with the $\mathrm{NH}_{3}{ }^{+}$group, two water molecules interacting with the $\mathrm{COO}^{-}$group and two more water molecules to complete the solvation shell. For these cases, the unit cell parameters of the BNNTs have been enlarged thrice to avoid lateral interactions between water molecules of adjacent unit cells. It is worth mentioning that a statistical sampling of the hypersurface of these systems can be carried out adopting either the molecular dynamics or the Monte Carlo approaches [41]. However, these calculations are extremely expensive at the ab-initio level adopting realistic models for BNNTs like the $(9,0)$ and the $(15,0)$ ones, which contain 129 and 201 atoms, respectively. For the present work, we have followed a different approach consisting of a progressive microsolvation of the dry interface at the Gly/BNNT structures. This microsolvation procedure consists of adding water molecules at the dry Gly/BNNT interface in such a way that Gly progressively loses direct contact with the BNNTs up to a point in which the interaction is fully bridged by water. This procedure has already been performed by some of us in other works for the interaction of Gly with silica [42] and hydroxyapatite [43] surfaces. Since the most stable state of Gly in water is the zwitterionic one, we considered the $\mathrm{BN} / \mathrm{zwitt}$ adducts as initial guesses for the progressive microsolvation. For the $(4,0)$, $(6,0)$ and $(9,0)$ BNNTs, the resulting structures are shown in Figure 2a. In the BN/CONH adduct, Gly directly interacts with the BNNTs in a similar fashion as in the gas-phase (i.e., $\mathrm{COO}^{-} \cdots \mathrm{B}_{\mathrm{BNNT}}$ dative bond and $\mathrm{NH}_{3}{ }^{+} \cdots \mathrm{N}_{\mathrm{BNNT}} \mathrm{H}$-bond, while the seven microsolvating water molecules are simple spectators interacting with available points of the $\mathrm{COO}^{-}$and $\mathrm{NH}_{3}{ }^{+}$groups through $\mathrm{H}$-bonding. It is worth remarking that now for the $(4,0)$ and $(6,0)$ BNNTs no $\mathrm{H}$ transfer from Gly to the BNNT occurs (at variance with the gas-phase adsorption, vide supra) due to the screening effect of water. The $\mathrm{BN} / \mathrm{CO}-\mathrm{w} / \mathrm{NH}$ and $\mathrm{BN} / \mathrm{NH}-\mathrm{w} / \mathrm{CO}$ adducts result from moving one spectator water molecule from the outer shell to the inner shell so that the following water mediated interactions $\mathrm{NH}_{3}{ }^{+} \cdots \mathrm{H}_{2} \mathrm{O} \cdots \mathrm{N}_{\mathrm{BNNT}}$ and $\mathrm{COO}^{-} \cdots \mathrm{H}_{2} \mathrm{O} \cdots \mathrm{B}_{\mathrm{BNNT}}$ occur respectively. Finally, From these two adducts, a second water displacement to remove the remaining direct Gly/BNNT interaction gives the $\mathrm{w} / \mathrm{CONH}$ adduct, in which water fully mediates the Gly/BNNT contact. It is worth mentioning that each of the seven $\mathrm{H}_{2} \mathrm{O}$ molecules can in principle be displaced from their positions to lead to a water mediated contact between Gly and BNNT. We choose the one exhibiting the weakest interaction energy with the other water molecules by computing the cost to remove one water molecule from the BN/CONH adduct by a single point energy evaluation for each $\mathrm{H}_{2} \mathrm{O}$.

For the $(15,0)$ BNNT, all the optimization calculations collapsed to structures with no direct contact between Gly and BNNT, the most stable one being presented in Figure 2b. In this structure, water fully 
solvates the Gly molecule and, at variance with the other BNNT cases, no charge transfer between water and BNNT takes place, due to the highly apolar character of this nanotube.

Figure 2. B3LYP-D2* optimized structures of the different calculated complexes for glycine interacting with the $(4,0),(6,0)$ and $(9,0)$ BNNTs (a) and with the $(15,0)$ BNNT (b) in the presence of seven water molecules. Distances in $\AA$ : bare values for the $(4,0)$ BNNT; values in parenthesis for the $(6,0)$ one; values in brackets for the $(9,0)$ one; and italic underscored values for the $(15,0)$ one. For this latter case, the distance is that between the $\mathrm{C}$ atom and the plane defined by the closest B-N hexagon ring.

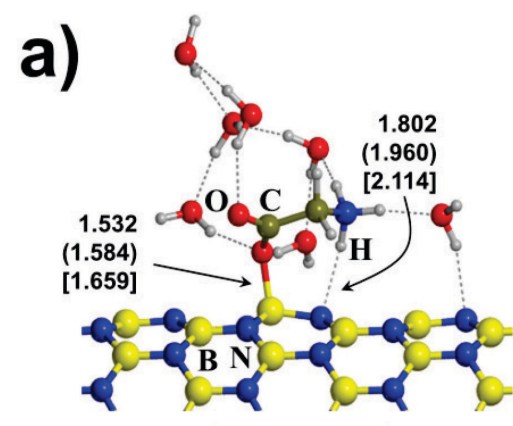

BN/CONH

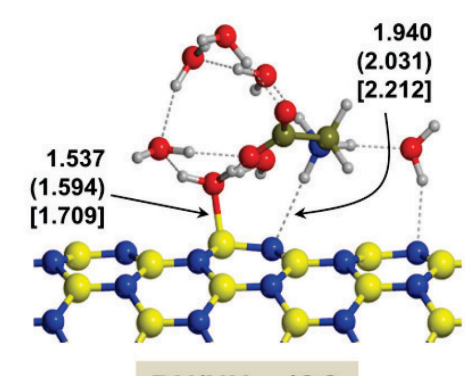

BN/NH-w/CO

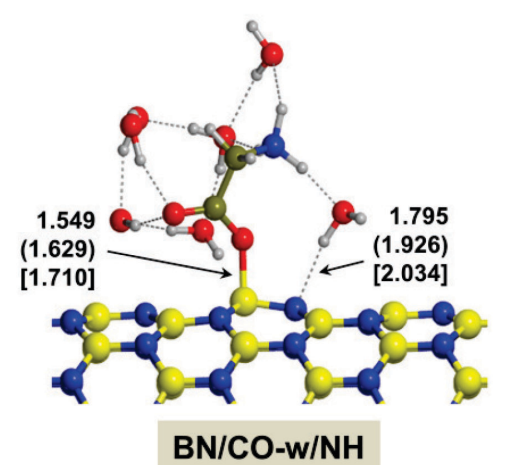

BN/CO-w/NH

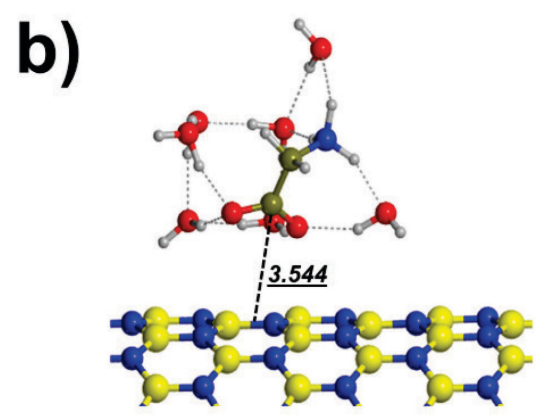

The relative stabilities between the different calculated adducts for a given microsolvated complex are shown in Table 3. As one can observe, for the $(6,0),(9,0)$ and $(15,0)$ BNNTs the most stable systems are the w/CONH adducts; i.e., those in which no direct Gly/BNNT contact occurs, whereas for the $(4,0)$ one, the $\mathrm{BN} / \mathrm{CO}-\mathrm{w} / \mathrm{NH}$ adduct (direct interaction only through the $\mathrm{COO}^{-}$) was found to be the most energetically stable one. It is worth mentioning, however, that the energy difference between the $\mathrm{BN}-\mathrm{CO}-\mathrm{w} / \mathrm{NH}$ and $\mathrm{w} / \mathrm{CONH}$ adducts for the $(4,0) \mathrm{BNNT}$ (the first and second most stable ones) is relatively small $\left(2.6 \mathrm{kcal} \mathrm{mol}^{-1}\right)$ and, accordingly, it might be inverted due to entropic effects associated with water rearrangement, as it is shown for peptide adsorption on hydrophobic and polar 
surfaces [44]. To further analyze this point, finite temperature molecular dynamics simulations would be desirable.

Table 3. Reaction energies $\left(\Delta E_{\mathrm{R} 1}, \Delta E_{\mathrm{R} 2}\right.$ and $\left.\Delta E_{\mathrm{R} 3}\right)$ and relative energies $\left(\Delta E_{\text {rel }}\right)$ of the formation of the Gly/7w/BNNT complexes. Values in units of kcal mol ${ }^{-1}$.

\begin{tabular}{llllll}
\hline System & Adduct & $\Delta \boldsymbol{E}_{\text {rel }}$ & $\Delta \boldsymbol{E}_{\mathbf{R} 1}$ & $\Delta \boldsymbol{E}_{\mathbf{R} 2}$ & $\Delta \boldsymbol{E}_{\mathbf{R} 3}$ \\
\hline Gly/7w/BNNT(4,0) & BN/CONH & 5.4 & -28.9 & -11.5 & 11.9 \\
& BN/CO-w/NH & 0.0 & -34.3 & -16.9 & 6.5 \\
& BN/NH-w/CO & 4.3 & -30.0 & -12.6 & 10.8 \\
& w/CONH & 2.6 & -31.7 & -14.3 & 9.1 \\
\hline Gly/7w/BNNT(6,0) & BN/CONH & 5.3 & -13.2 & -20.5 & 2.9 \\
& BN/CO-w/NH & 2.9 & -15.7 & -23.0 & 0.4 \\
& BN/NH-w/CO & 4.2 & -14.4 & -21.7 & 1.7 \\
& w/CONH & 0.0 & -18.6 & -25.9 & -2.5 \\
\hline Gly/7w/BNNT(9,0) & BN/CONH & 12.4 & -5.1 & -20.7 & 2.7 \\
& BN/CO-w/NH & 7.7 & -9.8 & -25.4 & -2.0 \\
& BN/NH-w/CO & 10.5 & -7.0 & -22.6 & 0.8 \\
& w/CONH & 0.0 & -17.5 & -33.1 & -9.7 \\
\hline Gly/7w/BNNT(15,0) & BN/CONH & - & - & - & - \\
& BN/CO-w/NH & - & - & - & - \\
& BN/NH-w/CO & - & - & - & - \\
& w/CONH & 0.0 & -12.8 & -31.4 & -8.1 \\
\hline
\end{tabular}

Besides these results, three different processes have moreover been considered to study the stability of the structures shown in Figure 2. The first one involves Gly solvated by $7 \mathrm{H}_{2} \mathrm{O}$ molecules being adsorbed on the clean walls of the BNNTs, whose reaction energy was computed as (reported by $\Delta E_{\mathrm{R} 1}$ of Table 2)

$$
\mathrm{Gly} / 7 \mathrm{w}+\mathrm{BNNT} \rightarrow \mathrm{Gly} / 7 \mathrm{w} / \mathrm{BNNT}
$$

where Gly/7w is glycine solvated by the seven $\mathrm{H}_{2} \mathrm{O}$ molecules and Gly/7w/BNNT represents the microsolvated complexes. The $\Delta E_{\mathrm{R} 1}$ column shows that for all BNNTs the process is exoenergetic, meaning that the structural rearrangement of the seven $\mathrm{H}_{2} \mathrm{O}$ molecules around Gly is compensated by the interaction with the BNNTs. Remarkably, limited to the most favorable adducts per BNNT, $\Delta E_{\mathrm{R} 1}$ values are less negative with increases in the nanotube radius, consistent with the less polar behavior of the BNNTs.

The second process envisages gas phase Gly adsorbed on the already microsolvated BNNTs by the seven water molecules, whereas the third one envisages the solvated Gly being adsorbed on the seven water hydrated BNNTs giving rise to the shown adducts with expulsion of seven water molecules (here considered as a H-bonded cluster), whose reaction energies were computed as (reported by $\Delta E_{\mathrm{R} 2}$ and $\Delta E_{\mathrm{R} 3}$ of Table 2)

$$
\begin{gathered}
\text { Gly }+7 \mathrm{w} / \mathrm{BNNT} \rightarrow \mathrm{Gly} / 7 \mathrm{w} / \mathrm{BNNT} \\
\mathrm{Gly} / 7 \mathrm{w}+7 \mathrm{w} / \mathrm{BNNT} \rightarrow \mathrm{Gly} / 7 \mathrm{w} / \mathrm{BNNT}+7 \mathrm{w}
\end{gathered}
$$


where $7 \mathrm{w} / \mathrm{BNNT}$ is the BNNT solvated by the seven $\mathrm{H}_{2} \mathrm{O}$ molecules and $7 \mathrm{w}$ the $\mathrm{H}$-bonded cluster made up by seven water molecules. These last two processes require the BNNTs in the presence of seven water molecules, whose optimized structures are given in Figure 3. The initial guess of these systems were the corresponding w/CONH adducts (no direct interaction between Gly and the BNNTs and accordingly the interaction between water molecules and BNNTs is maximum) in which the Gly molecule was removed. Consistent with the polar character of the BNNTs, several interactions between the water molecules and the $(4,0)$ BNNT via covalent dative and H-bond interactions take place, whereas by increasing the BNNT radius these interactions are progressively missed, up to the point in which for the $(15,0)$ no apparent interaction is observed.

Figure 3. B3LYP-D2* optimized structures of the different calculated complexes for the considered BNNTs in the presence of seven water molecules. Distances in $\AA$ : bare values for the $(4,0)$ BNNT; values in parenthesis for the $(6,0)$ one; values in brackets for the $(9,0)$ one; and italic underscored values for the $(15,0)$ one. For this latter case, the distance is that between the closest water $\mathrm{O}$ to the plane defined by the $\mathrm{B}-\mathrm{N}$ hexagon ring.

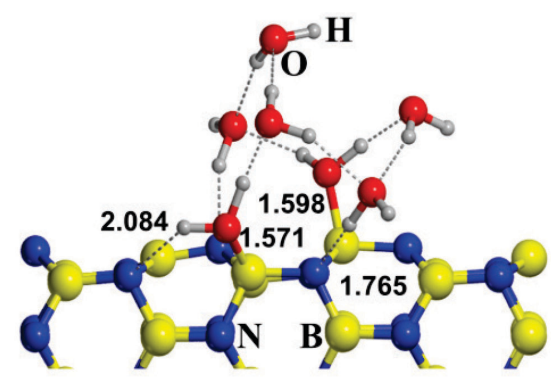

$7 w / B N N T(4,0)$

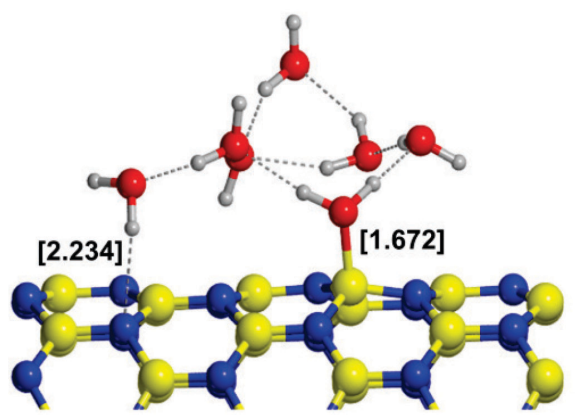

7w/BNNT(9,0)

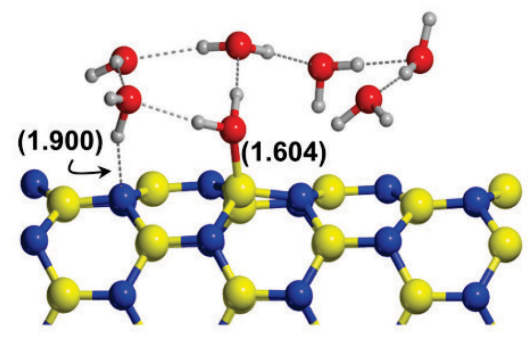

7 w/BNNT(6,0)

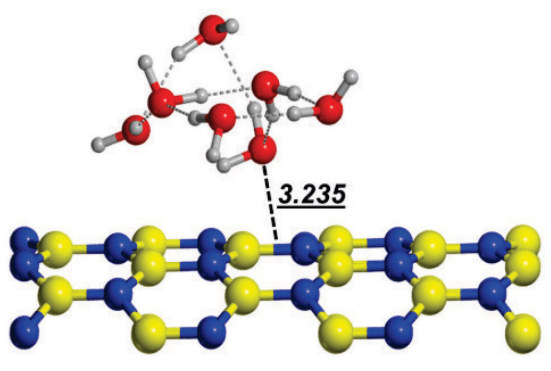

7w/BNNT(15,0)

The energetics of R2 is a tradeoff between the water affinity of the BNNTs and Gly (R2 is in essence the capture of the adsorbed water by Gly). The trend provided by the calculated $\Delta E_{\mathrm{R} 2}$ values indicates that R2 is more favorable when increasing the radius. That is, since the $(15,0)$ BNNT does not exhibit water affinity, the calculated $\Delta E_{\mathrm{R} 2}$ values are large and negative due to the strong interaction of water with Gly. In contrast, both the (4,0) BNNT and Gly exhibit large water affinity and accordingly the calculated $\Delta E_{\mathrm{R} 2}$ values are the less negative ones of the series. R3 is probably the most physically sound process as it involves the replacement of adsorbed water solvent by an already solvated Gly. Calculated $\Delta E_{\mathrm{R} 3}$ values indicate that such a water replacement is energetically favorable on the $(15,0)$ and $(9,0)$ BNNTs probably due to the low water affinity of these BNNTs. On the $(6,0)$ the process is still 
favorable by some amount but less than on the other two nanotubes $\left(\Delta E_{\mathrm{R} 3}=-2.5 \mathrm{kcal} \mathrm{mol}^{-1}\right)$, whereas on the $(4,0)$ calculated $\Delta E_{\mathrm{R} 1}$ was found to be positive, indicating that the overall interactions between Gly, water solvent and the nanotube are not as stable as the interaction between this nanotube and water.

\section{Computational Details}

All calculations were carried out using the periodic ab-initio code CRYSTAL09 [45]. All the SCF calculations and geometry optimizations were performed using the B3LYP-D* density functional method, which includes an empirical a posteriori correction term proposed by Grimme [46] to account for dispersion forces (missed in the pure B3LYP [47,48] method), but whose initial parameterization (D) was modified for extended systems ( $D^{*}$ ) [49], to provide accurate results for the calculations of cohesive energies of molecular crystals and of adsorption processes within a periodic treatment [49-51]. The adopted Gaussian functions consisted of an all electron triple- $\zeta$ 6-311G* standard basis set for the B and $\mathrm{N}$ atoms of the BNNTs and a TZP basis set from Ahlrichs and coworkers [52] for the atoms of Gly. This basis set combination has been proved to exhibit small basis set superposition error interaction energies $[18,50]$. The shrinking factor of the reciprocal space net defining the mesh of $\mathrm{k}$ points in the irreducible Brillouin zone was set to 5, which requires diagonalizing the Hamiltonian matrix in $3 \mathrm{k}$ points [53]. The accuracy of both Coulomb and exchange series was set to values of overlap integrals of $10^{-7}$ and $10^{-16}$, respectively, which ensure a very good numerical accuracy. A pruned $(75,974)$ grid has been used for the Gauss-Legendre and Lebedev quadrature schemes in the evaluation of functionals. The condition to achieve SCF convergence between two subsequent cycles was set to $10^{-7} \mathrm{Eh}$. Full relaxations of both lattice parameters and internal atomic coordinates by means of analytical energy gradients [54-56] have been carried out. The geometry optimization was performed by means of a quasi-Newton algorithm in which the quadratic step (BFGS Hessian updating scheme) is combined with a linear one (parabolic fit) [57].

\section{Conclusions}

Periodic quantum mechanical calculations have been used to simulate the adsorption of glycine (Gly) on different zig-zag $(n, 0)$ single-walled boron-nitride nanotubes (BNNTs, $n=4,6,9$ and 15) both in the gas-phase and in a microsolvated state (i.e., modeled by the presence of seven explicit water molecules) with the aim of determining the adsorption properties and the effect exerted by water as a function of surface curvature. These calculations are based on the B3LYP-D2* method, which includes the B3LYP hybrid functional with a revised version of the empirical a posteriori correction term (D2*) to account for dispersion interactions.

Gas-phase results clearly indicate that the most stable interaction between Gly and the $(4,0),(6,0)$ and $(9,0)$ BNNTs takes place through a covalent dative interaction between the $\mathrm{NH}_{2}$ group of Gly and the $\mathrm{B}$ atom of the BNNTs, which produce charge transfers from Gly to the BNNTs. In contrast, the interaction between Gly and the $(15,0)$ BNNT is mainly governed by non-covalent dispersive forces based on a $\pi$-stacking between the $\pi$ systems of the Gly $\mathrm{COOH}$ group and the B-N hexagon rings of the nanotube. Remarkably, the energy difference between these two adducts decreases when increasing the BNNT radius, in line with the polar/apolar character of the considered nanotubes. 
The adsorption of Gly on the BNNTs in the presence of seven water molecules has been studied adopting a progressive microsolvation procedure, in which water solvent molecules are added at the dry Gly/BNNT interfaces, hence progressively removing the direct interaction between Gly and the BNNTs. The obtained results indicate that for the $(6,0),(9,0)$ and $(15,0)$ BNNTs, the most stable microsolvated systems were found to exhibit no direct contact between the two partners; that is, the Gly/BNNT interfaces are fully bridged by the water solvent molecules. In contrast, for the $(4,0)$ one of the most stable systems shows direct contact between Gly and the BNNT through an interaction between the Gly $\mathrm{COO}^{-}$group and a nanotube $\mathrm{B}$ atom, although entropic effects (not accounted for in this work) might favor water mediated interface. Further energetic results point out that the larger the BNNT radius, the less water affinity. Accordingly, for larger radius BNNTs, the interaction between water and Gly was found to be predominant, in detriment to their interaction with the BNNT. However, it is found that the $(4,0)$ BNNT exhibits a large water affinity, which is reflected by the fact that the replacement of seven adsorbed water molecules by a microsolvated Gly has been found to be an unfavorable process.

The results presented here provide evidence that the adsorption properties of the BNNTs as well as their water affinity can significantly be modulated by controlling the tube diameter, as they are expected to exhibit different physico-chemical features, which may be of interest for the design of bioconjugated systems based on boron-nitride nanostructures and their potential bionanotechnological applications.

\section{Acknowledgments}

Albert Rimola is indebted to Ministry of Economy (MINECO) of the Spanish Government for a Juan de la Cierva contract. Financial support from MINECO (projects CTQ2013-40347-ERC and CTQ2011-24847/BQU) and from Department of Economy of the Catalan Government (project 2009SGR-638), and the use of the Catalonia Supercomputer Centre (CESCA) are gratefully acknowledged. Mariona Sodupe also acknowledges support through the 2011 ICREA Academia award.

\section{Author Contributions}

Simulations were carried out by Albert Rimola. Both Albert Rimola and Mariona Sodupe contributed to the discussion and writing of the paper.

\section{Conflicts of Interest}

The authors declare no conflict of interest.

\section{References}

1. Kujawa, P.; Winnik, F.M. Innovation in Nanomedicine through Materials Nanoarchitectonics. Langmuir 2013, 29, 7354-7361.

2. Wong, S.S.; Joselevich, E.; Woolley, A.T.; Cheung, C.L.; Lieber, C.M. Covalently functionalized nanotubes as nanometre- sized probes in chemistry and biology. Nature 1998, 394, 52-55. 
3. Aono, M.; Bando, Y.; Ariga, K. Nanoarchitectonics: Pioneering a New Paradigm for Nanotechnology in Materials Development. Adv. Mater. 2012, 24, 150-151.

4. Hong, R.; Fischer, N.O.; Verma, A.; Goodman, C.M.; Emrick, T.; Rotello, V.M. Control of Protein Structure and Function through Surface Recognition by Tailored Nanoparticle Scaffolds. J. Am. Chem. Soc. 2004, 126, 739-743.

5. You, C.-C.; Agasti, S.S.; De, M.; Knapp, M.J.; Rotello, V.M. Modulation of the Catalytic Behavior of $\alpha$-Chymotrypsin at Monolayer-Protected Nanoparticle Surfaces. J. Am. Chem. Soc. 2006, 128, 14612-14618.

6. Kang, Y.; Liu, Y.-C.; Wang, Q.; Shen, J.-W.; Wu, T.; Guan, W.-J. On the spontaneous encapsulation of proteins in carbon nanotubes. Biomaterials 2009, 30, 2807-2815.

7. Gao, H.; Kong, Y. Simulation of DNA-nanotube interactions. Annu. Rev. Mater. Res. 2004, 34, 123-150.

8. Chen, X.; Kis, A.; Zettl, A.; Bertozzi, C.R. A cell nanoinjector based on carbon nanotubes. Proc. Natl. Acad. Sci. USA 2007, 104, 8218-8222.

9. Chen, R.J.; Bangsaruntip, S.; Drouvalakis, K.A.; Wong Shi Kam, N.; Shim, M.; Li, Y.; Kim, W.; Utz, P.J.; Dai, H. Noncovalent functionalization of carbon nanotubes for highly specific electronic biosensors. Proc. Natl. Acad. Sci. USA 2003, 100, 4984-4989.

10. Chen, R.J.; Zhang, Y.; Wang, D.; Dai, H. Noncovalent Sidewall Functionalization of Single-Walled Carbon Nanotubes for Protein Immobilization. J. Am. Chem. Soc. 2001, 123, 3838-3839.

11. Cui, Y.; Wei, Q.; Park, H.; Lieber, C.M. Nanowire Nanosensors for Highly Sensitive and Selective Detection of Biological and Chemical Species. Science 2001, 293, 1289-1292.

12. Staii, C.; Johnson, A.T.; Chen, M.; Gelperin, A. DNA-Decorated Carbon Nanotubes for Chemical Sensing. Nano Lett. 2005, 5, 1774-1778.

13. Heller, D.A.; Jeng, E.S.; Yeung, T.-K.; Martinez, B.M.; Moll, A.E.; Gastala, J.B.; Strano, M.S. Optical Detection of DNA Conformational Polymorphism on Single-Walled Carbon Nanotubes. Science 2006, 311, 508-511.

14. Pantarotto, D.; Partidos, C.D.; Graff, R.; Hoebeke, J.; Briand, J.-P.; Prato, M.; Bianco, A. Synthesis, Structural Characterization, and Immunological Properties of Carbon Nanotubes Functionalized with Peptides. J. Am. Chem. Soc. 2003, 125, 6160-6164.

15. Tu, X.; Manohar, S.; Jagota, A.; Zheng, M. DNA sequence motifs for structure-specific recognition and separation of carbon nanotubes. Nature 2009, 460, 250-253.

16. Golberg, D.; Bando, Y.; Huang, Y.; Terao, T.; Mitome, M.; Tang, C.; Zhi, C. Boron Nitride Nanotubes and Nanosheets. ACS Nano 2010, 4, 2979-2993.

17. Golberg, D.; Bando, Y.; Tang, C.C.; Zhi, C.Y. Boron Nitride Nanotubes. Adv. Mater. 2007, 19, 2413-2432.

18. Rimola, A.; Sodupe, M. Physisorption vs. chemisorption of probe molecules on boron nitride nanomaterials: The effect of surface curvature. Phys. Chem. Chem. Phys. 2013, 15, 13190-13198.

19. Magrez, A.; Kasas, S.; Salicio, V.; Pasquier, N.; Seo, J.W.; Celio, M.; Catsicas, S.; Schwaller, B.; Forró, L. Cellular Toxicity of Carbon-Based Nanomaterials. Nano Lett. 2006, 6, 1121-1125. 
20. Chen, X.; Wu, P.; Rousseas, M.; Okawa, D.; Gartner, Z.; Zettl, A.; Bertozzi, C.R. Boron Nitride Nanotubes Are Noncytotoxic and Can Be Functionalized for Interaction with Proteins and Cells. J. Am. Chem. Soc. 2009, 131, 890-891.

21. Yu, J.; Chen, Y.; Cheng, B.M. Dispersion of boron nitride nanotubes in aqueous solution with the help of ionic surfactants. Solid State Commun. 2009, 149, 763-766.

22. Zhi, C.; Bando, Y.; Tang, C.; Golberg, D. Immobilization of Proteins on Boron Nitride Nanotubes. J. Am. Chem. Soc. 2005, 127, 17144-17145.

23. Gao, Z.; Zhi, C.; Bando, Y.; Golberg, D.; Serizawa, T. Isolation of Individual Boron Nitride Nanotubes via Peptide Wrapping. J. Am. Chem. Soc. 2010, 132, 4976-4977.

24. Zhi, C.; Bando, Y.; Wang, W.; Tang, C.; Kuwahara, H.; Golberg, D. DNA-Mediated Assembly of Boron Nitride Nanotubes. Chem. Asian J. 2007, 2, 1581-1585.

25. Ciofani, G.; Raffa, V.; Menciassi, A.; Dario, P. Preparation of Boron Nitride Nanotubes Aqueous Dispersions for Biological Applications. J. Nanosci. Nanotechnol. 2008, 8, 6223-6231.

26. Ciofani, G.; Raffa, V.; Menciassi, A.; Cuschieri, A. Cytocompatibility, interactions, and uptake of polyethyleneimine-coated boron nitride nanotubes by living cells: Confirmation of their potential for biomedical applications. Biotechnol. Bioeng. 2008, 101, 850-858.

27. Ciofani, G.; Danti, S.; D’Alessandro, D.; Moscato, S.; Menciassi, A. Assessing cytotoxicity of boron nitride nanotubes: Interference with the MTT assay. Biochem. Biophys. Res. Commun. 2010, 394, 405-411.

28. Ciofani, G.; Danti, S.; D’Alessandro, D.; Ricotti, L.; Moscato, S.; Bertoni, G.; Falqui, A.; Berrettini, S.; Petrini, M.; Mattoli, V.; et al. Enhancement of Neurite Outgrowth in Neuronal-Like Cells following Boron Nitride Nanotube-Mediated Stimulation. ACS Nano 2010, 4, 6267-6277.

29. Horváth, L.; Magrez, A.; Golberg, D.; Zhi, C.; Bando, Y.; Smajda, R.; Horváth, E.; Forró, L.; Schwaller, B. In Vitro Investigation of the Cellular Toxicity of Boron Nitride Nanotubes. ACS Nano 2011, 5, 3800-3810.

30. Akdim, B.; Kim, S.N.; Naik, R.R.; Maruyama, B.; Pender, M.J.; Pachter, R. Understanding effects of molecular adsorption at a single-wall boron nitride nanotube interface from density functional theory calculations. Nanotechnology 2009, 20, 355705.

31. Saikat, M.; Gowtham, S.; Ralph, H.S.; Ravindra, P.; Shashi, P.K. Theoretical study of physisorption of nucleobases on boron nitride nanotubes: A new class of hybrid nano-biomaterials. Nanotechnology 2010, 21, 165703.

32. Lin, Q.; Zou, X.; Zhou, G.; Liu, R.; Wu, J.; Li, J.; Duan, W. Adsorption of DNA/RNA nucleobases on hexagonal boron nitride sheet: An ab initio study. Phys. Chem. Chem. Phys. 2011, $13,12225-12230$.

33. Wu, X.; An, W.; Zeng, X.C. Chemical Functionalization of Boron-Nitride Nanotubes with $\mathrm{NH}_{3}$ and Amino Functional Groups. J. Am. Chem. Soc. 2006, 128, 12001-12006.

34. Mukhopadhyay, S.; Scheicher, R.H.; Pandey, R.; Karna, S.P. Sensitivity of Boron Nitride Nanotubes toward Biomolecules of Different Polarities. J. Phys. Chem. Lett. 2011, 2, 2442-2447.

35. Yang, C.-K. Exploring the interaction between the boron nitride nanotube and biological molecules. Comput. Phys. Commun. 2011, 182, 39-42. 
36. Noel, Y.; D’Arco, P.; Demichelis, R.; Zicovich-Wilson, C.M.; Dovesi, R. On the Use of Symmetry in the Ab Initio Quantum Mechanical Simulation of Nanotubes and Related Materials. J. Comput. Chem. 2010, 31, 855-862.

37. Demichelis, R.; Noel, Y.; D’Arco, P.; Rerat, M.; Zicovich-Wilson, C.M.; Dovesi, R. Properties of Carbon Nanotubes: An ab Initio Study Using Large Gaussian Basis Sets and Various DFT Functionals. J. Phys. Chem. C 2011, 115, 8876-8885.

38. D’Arco, P.; Noel, Y.; Demichelis, R.; Dovesi, R. Single-layered chrysotile nanotubes: A quantum mechanical ab initio simulation. J. Chem. Phys. 2009, 131, doi:10.1063/1.3251791.

39. Demichelis, R.; Noel, Y.; D’Arco, P.; Maschio, L.; Orlando, R.; Dovesi, R. Structure and energetics of imogolite: A quantum mechanical ab initio study with B3LYP hybrid functional. J. Mater. Chem. 2010, 20, 10417-10425.

40. Ferrari, A.M.; Szieberth, D.; Zicovich-Wilson, C.M.; Demichelis, R. Anatase(001) 3 ML Nanotubes, The First $\mathrm{TiO}_{2}$ Nanotube With Negative Strain Energies: A DFT Prediction. J. Phys. Chem. Lett. 2010, 1, 2854-2857.

41. Marx, D.; Hutter, J. Ab Initio Molcular Dynamics: Theory and Implementation. In Modern Methods and Algorithms of Quantum Chemistry; Grotendorst, J., Ed.; Forschungszentrum Jülich: Jülich, Germany, 2000.

42. Rimola, A.; Civalleri, B.; Ugliengo, P. Neutral vs. Zwitterionic Glycine Forms at the Water/Silica Interface: Structure, Energies, and Vibrational Features from B3LYP Periodic Simulations. Langmuir 2008, 24, 14027-14034.

43. Rimola, A.; Corno, M.; Zicovich-Wilson, C.M.; Ugliengo, P. Ab initio modeling of protein/biomaterial interactions: Competitive adsorption between glycine and water onto hydroxyapatite surfaces. Phys. Chem. Chem. Phys. 2009, 11, 9005-9007.

44. Schwierz, N.; Horinek, D.; Liese, S.; Pirzer, T.; Balzer, B.N.; Hugel, T.; Netz, R.R. On the Relationship between Peptide Adsorption Resistance and Surface Contact Angle: A Combined Experimental and Simulation Single-Molecule Study. J. Am. Chem. Soc. 2012, 134, 19628-19638.

45. Dovesi, R.; Saunders, V.R.; Roetti, C.; Orlando, R.; Zicovich-Wilson, C.M.; Pascale, F.; Civalleri, B.; Doll, K.; Harrison, N.M.; Bush, I.J.; et al. CRYSTAL09 User's Manual; University of Torino: Torino, Italy, 2009; p. 307.

46. Grimme, S. Semiempirical GGA-type density functional constructed with a long-range dispersion correction. J. Comput. Chem. 2006, 27, 1787-1799.

47. Becke, A.D. Density-functional thermochemistry. III. The role of exact exchange. J. Chem. Phys. 1993, 98, 5648-5652.

48. Lee, C.; Yang, W.; Parr, R.G. Development of the Colle-Salvetti correlation-energy formula into a functional of the electron density. Phys. Rev. B 1988, 37, 785-789.

49. Civalleri, B.; Zicovich-Wilson, C.M.; Valenzano, L.; Ugliengo, P. B3LYP augmented with an empirical dispersion term (B3LYP-D*) as applied to molecular crystals. CrystEngComm 2008, 10, 405-410.

50. Rimola, A.; Civalleri, B.; Ugliengo, P. Physisorption of aromatic organic contaminants at the surface of hydrophobic/hydrophilic silica geosorbents: A B3LYP-D modeling study. Phys. Chem. Chem. Phys. 2010, 12, 6357-6366. 
51. Civalleri, B.; Maschio, L.; Ugliengo, P.; Zicovich-Wilson, C.M. Role of dispersive interactions in the $\mathrm{CO}$ adsorption on $\mathrm{MgO}$ (001): Periodic B3LYP calculations augmented with an empirical dispersion term. Phys. Chem. Chem. Phys. 2010, 12, 6382-6386.

52. Schafer, A.; Horn, H.; Ahlrichs, R. Fully optimized contracted Gaussian basis sets for atoms Li to Kr. J. Chem. Phys. 1992, 97, 2571-2577.

53. Monkhorst, H.J.; Pack, J.D. Special points for Brillouin-zone integrations. Phys. Rev. B 1976, 13, 5188-5192.

54. Doll, K.; Saunders, V.R.; Harrison, N.M. Analytical Hartree-Fock gradients for periodic systems. Int. J. Quantum Chem. 2001, 82, 1-13.

55. Doll, K. Implementation of analytical Hartree-Fock gradients for periodic systems. Comput. Phys. Commun. 2001, 137, 74-88.

56. Doll, K.; Dovesi, R.; Orlando, R. Analytical Hartree-Fock gradients with respect to the cell parameter for systems periodic in three dimensions. Theor. Chem. Acc. 2004, 112, 394-402.

57. Civalleri, B.; D’Arco, P.; Orlando, R.; Saunders, V.R.; Dovesi, R. Hartree-Fock geometry optimisation of periodic systems with the CRYSTAL code. Chem. Phys. Lett. 2001, 348, $131-138$.

(C) 2014 by the authors; licensee MDPI, Basel, Switzerland. This article is an open access article distributed under the terms and conditions of the Creative Commons Attribution license (http://creativecommons.org/licenses/by/3.0/). 